\title{
artigo
}

Sousa, T.J.; Oliveira, D.F.; Santos, A.C.G.; Conceição, L.N.; Estrela, F.M.; Marinho, C.S.; David, E.A.R.;

Medidas de prevenção relacionadas aos eventos adversos na unidade de terapia intensiva

\section{Medidas de prevenção relacionadas aos eventos adversos na unidade de terapia intensiva}

\author{
Prevention measures related to adverse events in the intensive care unit \\ Medidas de prevención relacionadas con eventos adversos en la unidad de cuidados intensivos
}

\begin{abstract}
RESUMO
Os eventos adversos representam um grave problema na assistência de saúde na unidade de terapia intensiva, impactando diretamente no tempo de internação hospitalar e mortalidade dos pacientes. Objetivo: Identificar medidas de prevenção relacionadas aos eventos adversos na unidade de terapia intensiva por meio de revisão de literatura Método: Revisão integrativa da literatura, realizada nas bases de dados: Scientific Eletronic Library Online e Literatura Latino-Americana e do Caribe em Ciências da Saúde e Google acadêmico, publicados entre 2014 a 2020, utilizado os descritores: Medidas preventivas, eventos adversos, unidade de terapia intensiva e segurança do paciente. Resultados: doze artigos foram selecionados tendo como categorias da discussão: Eventos adversos com maior taxa de incidência na unidade de terapia intensiva, fatores relacionados a ocorrência de eventos adversos na unidade de terapia intensiva e medidas de prevenção destes eventos adversos. Conclusão: Para prevenção dos eventos adversos, devem ser implementadas, estratégias pelos gestores de enfermagem visando melhoria da assistência, redução de sobrecarga de trabalho dos profissionais, registros completos em prontuários eletrônicos, educação continuada, comunicação efetiva entre equipe multiprofissional, estabelecimento de normas institucionais e minimização de falhas assistenciais.
\end{abstract}

DESCRITORES: Medidas preventivas; Eventos adversos; Unidade de Terapia Intensiva; Segurança do paciente.

\section{ABSTRACT}

Adverse events represent a serious problem in health care in the intensive care unit, directly impacting the length of hospital stay and mortality of patients. Objective: Identify preventive measures related to adverse events in the intensive care unit through literature review Method: Integrative literature review, carried out in the databases: Scientific Eletronic Library Online and Latin American and Caribbean Literature in Health Sciences and Google academic, published between 2014 to 2020, used the descriptors: Preventive measures, adverse events, intensive care unit and patient safety Results: twelve articles were selected with the following discussion categories: Adverse events with a higher incidence rate in the intensive care unit, factors related to the occurrence of adverse events in the intensive care unit and preventive measures for these adverse events. Conclusion: To prevent adverse events, strategies should be implemented by nursing managers to improve care, reduce work overload for professionals, complete records in electronic medical records, continuing education, effective communication between the multidisciplinary team, establishment of institutional norms and minimization of care failures.

DESCRIPTORS: Preventive measures; Adverse events; Intensive care unit; Patient safety.

\section{RESUMEN}

Los eventos adversos representan un problema grave en la atención de la salud en la unidad de cuidados intensivos, impactando directamente en la duración de la estancia hospitalaria y la mortalidad de los pacientes. Objetivo: Identify preventive measures related to adverse events in the intensive care unit through literature review Método: Revisión integrativa de la literatura, realizada en las bases de datos: Scientific Eletronic Library Online y Literatura Latinoamericana y del Caribe en Ciencias de la Salud y académica de Google, publicada entre 2014 a 2020, descriptores utilizados: medidas preventivas, eventos adversos, unidad de cuidados intensivos y seguridad del paciente. Resultados: se seleccionaron doze artículos, con las siguientes categorías de discusión: Eventos adversos con mayor tasa de incidencia en la unidad de cuidados intensivos, factores relacionados con la ocurrencia de eventos adversos en la unidad de cuidados intensivos y medidas preventivas para estos eventos adversos. Conclusión: Para prevenir eventos adversos se deben implementar estrategias por parte de los gerentes de enfermería para mejorar la atención, reducir la sobrecarga de trabajo de los profesionales, completar registros en historias clínicas electrónicas, educación continua, comunicación efectiva entre el equipo multidisciplinario, establecimiento de normas institucionales y minimización de fallas en la atención.

DESCRIPTORES: Medidas preventivas; Eventos adversos; Unidad de terapia intensiva; Seguridad del paciente.

RECEBIDO EM: 29/01/2021 APROVADO EM: 10/02/2021 
Tamires Jesus Sousa

Enfermeira. Mestranda pela Universidade Federal da Bahia.

ORCID: 0000-0003-4141-9097

\section{Daniela Fagundes de Oliveira}

Enfermeira. Mestranda da Universidade Federal da Bahia.

ORCID: 0000-0003-4804-7257

\section{Amanda Cibele Gaspar dos Santos}

Enfermeira. Especialista em enfermagem Oncológica; Mestranda da Universidade Federal da Bahia. Docente do Centro Universitário Maurício de Nassau.

ORCID: 0000-0001-9477-3757

\section{Leilane Nascimento da Conceição}

Enfermeira. Membro da Comissão de Pele das Obras Sociais Irmã Dulce.

ORCID: 0000-0003-2161-7563

\section{Fernanda Matheus Estrela}

Enfermeira. Doutora em Enfermagem e Saúde. Docente da Universidade Estadual de Feira de Santana.

ORCID: 0000-0001-7501-6187

\section{Cláudia Silva Marinho}

Enfermeira. Doutora em Ciências. Docente da Universidade Federal da Bahia.

ORCID: 0000-0002-0597-8126

\section{Rose Ana Rios David}

Enfermeira. Doutora em Enfermagem. Docente da Universidade Federal da Bahia.

ORCID: 0000-0003-1316-2394

\section{INTRODUÇÃO}

A Agência Nacional de Vigilância Sanitária (Anvisa) ${ }^{(1)}$, em 2004, classificou evento adverso (EA) como um incidente que resulta em prejuízo à saúde, podendo causar comprometimentos do corpo e/ou algum efeito posterior, incluindo patologias, lesão, dor, falecimento, incapacidade ou disfunção, podendo ser físico, social ou psíquico.

Os eventos adversos representam um grave problema na assistência de saúde, especialmente na unidade de terapia intensiva (UTI), que os pacientes apresentam estados de saúde mais graves, impactando diretamente no aumento do tempo de internação hospitalar e da mortalidade dos pacientes ${ }^{(2)}$. Sua ocorrência está relacionada a prática dos profissionais de saúde, como à falta de atenção no preparo e administração de medicamentos e à negligência dos profissionais em relação aos cuidados prestados durante a assistência ${ }^{(3)}$. Sendo imprescindível que os profissionais de saúde mante-
Sua ocorrência

está relacionada a

prática dos profissionais

de saúde, comoà falta

de atenção no preparo

e administração de

medicamentos e

à negligência dos

profissionais em relação

aos cuidados prestados

durante a assistência. nham-se atualizados nos procedimentos técnicos de enfermagem, através da realização de cursos de capacitação e treinamentos no ambiente de trabalho.

De acordo com a Agência Nacional de Vigilância Sanitária (ANVISA), resolução RDC n³6, constitui que a segurança do paciente é definida como a redução, ao mínimo aceitável, do risco de dano desnecessário associado à atenção à saúde ${ }^{(1)}$. É componente importante na prestação de assistência com qualidade.

Esforços do governo têm orientado boas práticas para minimizar os EAs como o Programa Nacional de Segurança do Paciente (PNSP) que foi instituído através da portaria nº $529 / 13$ do Ministério da Saúde (MS) e visa contribuir para a qualificação do cuidado em saúde em todos os estabelecimentos de saúde, também a Resolução da diretoria colegiada (RDC) $36 / 2013$ que institui ações para a segurança do paciente nos serviços de saúde.

A segurança do paciente é um grande desafio para os cuidados de saúde atual- 


\section{artigo}

Sousa, T.J.; Oliveira, D.F.; Santos, A.C.G.; Conceição, L.N.; Estrela, F.M.; Marinho, C.S.; David, E.A.R.

Medidas de prevenção relacionadas aos eventos adversos na unidade de terapia intensiva

mente. Admitir a ocorrência de erros ou acidentes adversos com consequências sérias aos pacientes estão levando os gestores de saúde, equipe de enfermagem e equipe médica a procurarem alternativas para reduzirem as situações de riscos nas instituições hospitalares ${ }^{(4)}$.

Portanto, este estudo evidencia a importância de abordar as medidas de prevenção dos eventos adversos na Unidade de Terapia Intensiva (UTI) tendo em vista que a qualidade na assistência prestada pela enfermagem está diretamente associada à segurança do paciente e que os profissionais de enfermagem estão frequentemente expostos a ocorrência de eventos adversos. Esta pesquisa foi elaborada devido a necessidade de compilar os achados na literatura evidenciando lacuna acerca da temática abordada.
Diante disso, emergiu como questão de pesquisa: Quais as medidas preventivas para reduzir a ocorrência de eventos adversos na UTI? Deste modo, tem como objetivo geral: identificar na literatura medidas de prevenção relacionadas aos eventos adversos na UTI e como objetivos específicos: identificar os eventos adversos com maior ocorrência na UTI por meio de revisão integrativa da literatura.

\section{MÉTODO}

Para responder tal questão de pesquisa, o presente estudo se configura como sendo uma uma revisão integrativa da literatura com a finalidade de conhecer as medidas de prevenção dos eventos adversos na UTI.

Estudo de revisão integrativa da literatura, à qual se propõe ao apontamento de

\section{Quadro 1 - Aplicação da estratégia PICo}

\begin{tabular}{|c|c|c|}
\hline ESTRATÉGIA & DEFINIÇÃo & APLICAÇÃo \\
\hline P & Problema & Eventos adversos \\
\hline I & Intervenção & Medidas de Prevenção \\
\hline Co & Contexto & UTI \\
\hline
\end{tabular}

Figura 1 - Fluxograma de apresentação do processo inclusão e exclusão dos estudos, PRISMA.

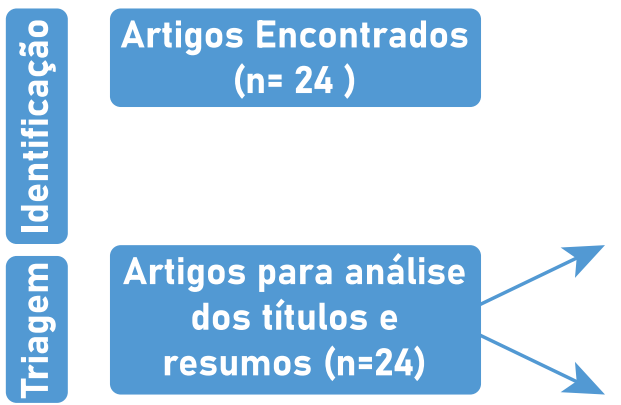

Artigos completos analisados $(n=13)$

Artigos excluídos após análise dos títulos e resumos $(\mathbf{n = 1 0})$

Artigos excluídos por duplicidade $(n=1)$

Artigos excluídos por não atenderem 0 objetivo do estudo $(n=1)$

Artigos incluídos $(n=12)$

lacunas no conhecimento, fornecendo ao pesquisador o direcionamento aos temas que precisam de exploração científica ${ }^{(6)}$. Esta pesquisa foi realizada conforme as seis etapas preconizadas e em conformidade com a Revised Standards for Quality Improvement Reporting Excellence (SQUIRE 2.0) ${ }^{(7)}$.

Para a primeira etapa, utilizou-se a estratégia PICo que representa um acrônimo para Problema (P), Intervenção (I), Contexto (Co), sendo esses, elementos da questão de pesquisa e da construção da pergunta orientadora do estudo, conforme apontado no quadro $1^{(8)}$. Desse modo, tendo como base tal estruturação, a pesquisa foi guiada pela seguinte questão: Quais as medidas de prevenção dos eventos adversos na UTI.

Foi realizado um levantamento nas bases de dados eletrônicos Scientific Eletronic Library Online (Scielo) e Literatura Latino-Americana e do Caribe em Ciências da Saúde (LILACS) e Google acadêmico, a busca foi realizada nos meses de Agosto a Dezembro de 2020, utilizando os seguintes descritores DECs: Medidas preventivas; eventos adversos; Unidade de Terapia Intensiva, segurança do paciente;

Realizando a sistematização dos dados através da técnica de análise de conteúdos utilizou-se o boleano "AND" uma vez que o mesmo favorece a intersecção no decorrer das pesquisas.

Realizou-se a leitura dos títulos e resumos e adotou-se como critérios de inclusão artigos originais em Português, publicados na íntegra entre os anos de 2014 a 2020 que abordassem à temática do estudo. Foram excluídos os artigos duplicados, que não abordaram o tema do estudo e os que ficaram fora da delimitação temporal. Essa delimitação se deve a manutenção dos dados atualizados.

Foram encontrados 24 (vinte e quatro) artigos, em que destes: 20 (vinte) da base de dados Scielo, 2 (dois) da base de dados Lilacs e 2 (dois) do google acadêmico. Destes 10 (dez) artigos foram excluídos após a análise dos títulos e resumos por não atenderem a temática abordada o e 3 (três) após leitura na íntegra por fuga do tema e 1 (um) por duplicidade. Resultan-

5942 saúdecoletiva = 2021; (11) N.65 
do em 10 (Dez) artigos que compuseram este estudo.

\section{RESULTADOS}

Foram encontrados 12 artigos para compor o presente trabalho e correspondiam ao tema do presente trabalho entre o ano de 2014 a 2020, na base de dados da SCIELO, Google acadêmico e BDENF.

Diante o exposto, os artigos foram separados por ano de publicação, sendo sub- metidos a uma leitura de forma criteriosa e registrados. O quadro 1, apresenta a síntese dos estudos analisados, conforme: autores e ano, título, periódico, objetivo e resultados. Os estudos foram organizados em ordem crescente do ano de 2014 a 2019.

Quadro 1 - Epítome dos artigos selecionados ( $\mathrm{n=12)}$ quanto aos autores e ano, periódico, título, objetivo e resultados. Salvador. Bahia, Brasil, 2020.

\begin{tabular}{|c|c|c|c|}
\hline AUTORES/ANO & TÍTULO/ PERIÓDICO & OBJETIVO & RESULTADOS \\
\hline $\begin{array}{l}\text { Barbosa TP, Oliveira } \\
\text { GAA, Lopes MNA, } \\
\text { Poletti NAA, Becca- } \\
\text { ria LM./ } 2014^{(9)}\end{array}$ & $\begin{array}{l}\text { Práticas assistências para } \\
\text { segurança do paciente em } \\
\text { unidade de terapia intensiva } \\
\text { Acta paul. enferm. }\end{array}$ & $\begin{array}{c}\text { Objetivo: Verificar as boas prá- } \\
\text { ticas assistenciais de enferma- } \\
\text { gem para segurança do paciente } \\
\text { em unidade de terapia intensiva. }\end{array}$ & $\begin{array}{l}\text { Em conjunto, as boas práticas estão sendo } \\
\text { realizadas com índice acima de } 90 \% \text {, com } \\
\text { exceção da mudança de decúbito, restrições de } \\
\text { membros limpas e circuito do ventilador }\end{array}$ \\
\hline $\begin{array}{l}\text { Novaretti MCZ, } \\
\text { Santos EV, Quitério } \\
\text { LM, Daud-Gallotti } \\
\text { RM./ } 2014^{(10)}\end{array}$ & $\begin{array}{l}\text { Sobrecarga de trabalho da } \\
\text { Enfermagem e incidentes } \\
\text { e Eventos adversos em } \\
\text { pacientes internados em } \\
\text { UTI Rev. Bras Enferm. }\end{array}$ & $\begin{array}{l}\text { Identificar a influência da } \\
\text { sobrecarga de trabalho da } \\
\text { Enfermagem na ocorrência de } \\
\text { incidentes sem lesão e eventos } \\
\text { adversos em } 399 \text { pacientes } \\
\text { internados em Unidades de } \\
\text { Terapia Intensiva (UTI) }\end{array}$ & $\begin{array}{l}\text { Nessas admissões, aproximadamente } 78 \% \\
\text { dos incidentes sem lesão e eventos adversos } \\
\text { em pacientes foram relacionados à esfera da } \\
\text { Enfermagem. Estas guardam relação com a } \\
\text { sobrecarga de trabalho, aumentaram o número } \\
\text { de dias de internação e o risco de óbito dos } \\
\text { pacientes estudados. }\end{array}$ \\
\hline $\begin{array}{c}\text { Souza RF, Alves AS, } \\
\text { Alencar IGM/ } 2018 \\
\qquad 9^{(11)}\end{array}$ & $\begin{array}{l}\text { Eventos adversos na unida- } \\
\text { de de terapia intensiva Rev. } \\
\text { enferm. UFPE online }\end{array}$ & $\begin{array}{l}\text { Caracterizar os eventos } \\
\text { adversos de uma Unidade de } \\
\text { Terapia Intensiva }\end{array}$ & $\begin{array}{l}\text { Constatou-se a ocorrência de } 152 \text { eventos } \\
\text { adversos. O perfil dos pacientes destacou-se } \\
\text { por ser do sexo masculino, adultos jovens, com } \\
\text { média de } 45 \text { anos. }\end{array}$ \\
\hline Costa TD/ $2015^{(12)}$ & $\begin{array}{l}\text { Avaliação do cuidado de } \\
\text { enfermagem na pers- } \\
\text { pectiva da segurança do } \\
\text { paciente em Unidade de } \\
\text { Terapia Intensiva: na visão } \\
\text { de profissionais, pacientes } \\
\text { e familiares Universidade } \\
\text { Federal do Rio Grande do } \\
\text { Norte }\end{array}$ & $\begin{array}{l}\text { Avaliar o cuidado de enferma- } \\
\text { gem na perspectiva da seguran- } \\
\text { ça do paciente em Unidades de } \\
\text { Terapia Intensiva. }\end{array}$ & $\begin{array}{c}\text { Os resultados da análise da "estrutura" e "pro- } \\
\text { cessos" demonstrou que a maioria dos achados } \\
\text { se encontrava fora dos padrões de adequação, } \\
\text { o que aponta condições precárias nas estru- } \\
\text { turas e, processos incipientes nos serviços de } \\
\text { saúde. }\end{array}$ \\
\hline $\begin{array}{l}\text { Roque KE, Tonini T, } \\
\text { Melo ECP/ } 2016^{(2)}\end{array}$ & $\begin{array}{l}\text { Eventos adversos na uni- } \\
\text { dade de terapia intensiva: } \\
\text { impacto na mortalidade e } \\
\text { no tempo de internação } \\
\text { em um estudo prospectivo } \\
\text { Revista Científica Fac Mais }\end{array}$ & $\begin{array}{l}\text { Avaliar a ocorrência de eventos } \\
\text { adversos e o impacto deles } \\
\text { sobre o tempo de permanência } \\
\text { e a mortalidade na unidade de } \\
\text { terapia intensiva (UTI). }\end{array}$ & $\begin{array}{l}\text { Confirmados } 324 \text { eventos adversos em } 115 \\
\text { pacientes internados ao longo de um ano de } \\
\text { seguimento. A taxa de incidência foi de 9,3 } \\
\text { eventos adversos por } 100 \text { pacientes-dia. }\end{array}$ \\
\hline $\begin{array}{l}\text { Minuzzi AP, Salum } \\
\text { NC, Locks MOH, } \\
\text { Amante LN, Matos } \\
\text { E./ 2016 }\end{array}$ & $\begin{array}{l}\text { Contribuiç̧ões da equipe de } \\
\text { saúde visando a promoção } \\
\text { da segurança do paciente } \\
\text { no cuidado intensivo Esc. } \\
\text { Anna Nery }\end{array}$ & $\begin{array}{l}\text { Apresentar as recomendações } \\
\text { dos profissionais de saúde de } \\
\text { uma Unidade de Terapia Intensi- } \\
\text { va para a melhoria da cultura de } \\
\text { segurança do paciente. }\end{array}$ & $\begin{array}{l}\text { Obteve-se } 124 \text { recomendações que foram } \\
\text { categorizadas de acordo com os aspectos do } \\
\text { instrumento. Destacando-se recomendações } \\
\text { relacionadas ao apoio da gestão hospitalar } \\
\text { para a segurança do paciente, em especial, ao } \\
\text { suprimento de recursos materiais; }\end{array}$ \\
\hline $\begin{array}{l}\text { Grilo PK, Barbosa } \\
\text { RL, Andolhe R, } \\
\text { Oliveira EM, Ducci } \\
\text { AJ, Bregalda RS et } \\
\text { al./ } 2017^{(14)}\end{array}$ & $\begin{array}{l}\text { Carga de trabalho de enfer- } \\
\text { magem, estresse/Burnout, } \\
\text { satisfação e incidentes em } \\
\text { unidade de terapia intensiva } \\
\text { de trauma Texto contexto - } \\
\text { enferm }\end{array}$ & $\begin{array}{l}\text { Avaliar a ocorrência de eventos } \\
\text { adversos relacionados a carga } \\
\text { de trabalho de enfermagem, } \\
\text { estresse/Burnout e satisfação. }\end{array}$ & $\begin{array}{l}\text { Ocorreram } 1.586 \text { incidentes, predominante- } \\
\text { mente incidentes sem dano (78,44\%). Entre a } \\
\text { equipe de enfermagem, } 77,40 \% \text { tinham niveis } \\
\text { médios de estresse; } 17,00 \% \text { apresentaram Bur- } \\
\text { nout; } 56,6 \% \text { estavam insatisfeitos e considera- } \\
\text { ram as características ambientais inadequadas. }\end{array}$ \\
\hline
\end{tabular}




\section{artigo}

Sousa, T.J.; Oliveira, D.F.; Santos, A.C.G.; Conceição, L.N.; Estrela, F.M.; Marinho, C.S.; David, E.A.R.;

Medidas de prevenção relacionadas aos eventos adversos na unidade de terapia intensiva

\begin{tabular}{|c|c|c|c|}
\hline $\begin{array}{l}\text { Silva R, Amante LN, } \\
\text { Salum NC, Girondi } \\
\text { JBR, Sebold LF./ } \\
2018^{(15)}\end{array}$ & $\begin{array}{l}\text { Incidentes e eventos adver- } \\
\text { sos no transporte intra hos- } \\
\text { pitalar em terapia intensiva } \\
\text { Revista de Enfermagem do } \\
\text { Centro-Oeste Mineiro }\end{array}$ & $\begin{array}{l}\text { Identificar os incidentes e } \\
\text { eventos adversos no transpor- } \\
\text { te intra-hospitalar em terapia } \\
\text { intensiva. }\end{array}$ & $\begin{array}{l}\text { No período, foram realizados } 1.559 \text { transpor- } \\
\text { tes intra-hospitalares, em } 1.348 \text { pacientes, } \\
\text { Eventos clínicos ocorreram em } 117 \text { transportes } \\
(7,5 \%) \text { e não clínicos em } 125 \text { transportes }(8,0 \%) \text {. }\end{array}$ \\
\hline Batista CF/ $2018^{(16)}$ & $\begin{array}{l}\text { Fatores que influenciam a } \\
\text { ocorrência de eventos ad- } \\
\text { versos Incidentes e eventos } \\
\text { adversos no transporte } \\
\text { intra hospitalar em terapia } \\
\text { intensiva na unidade de } \\
\text { terapia intensiva Rev. Eletr. } \\
\text { Evid \& Enferm }\end{array}$ & $\begin{array}{l}\text { Analisar publicações relaciona- } \\
\text { das aos fatores que influenciam } \\
\text { na ocorrência de eventos adver- } \\
\text { sos (EA) em Unidade de Terapia } \\
\text { Intensiva (UTI) adulta }\end{array}$ & $\begin{array}{c}\text { A não participação efetiva de gestores, e } \\
\text { demais membros da equipe interdisciplinar são } \\
\text { fatores que influenciam na ocorrência de EA, } \\
\text { outro fator importante é a carga de trabalho } \\
\text { excessiva e número reduzido na equipe de } \\
\text { enfermagem. }\end{array}$ \\
\hline $\begin{array}{l}\text { Cruz FF, Gonçalves } \\
\text { RP, Raimundo SR, } \\
\text { Amaral MS./ } 2018 \\
\qquad \text { (17) }\end{array}$ & $\begin{array}{l}\text { Segurança do paciente na } \\
\text { uti: uma revisão da literatu- } \\
\text { ra Revista Científica FacMais }\end{array}$ & $\begin{array}{l}\text { Apontar os principais even- } \\
\text { tos adversos/erros que são } \\
\text { cometidos na UTI e as medidas } \\
\text { recomendadas para os profis- } \\
\text { sionais da enfermagem que } \\
\text { visam garantir a segurança do } \\
\text { paciente em UTI. }\end{array}$ & $\begin{array}{l}\text { Percebeu-se que os principais erros e falhas } \\
\text { que ocorrem dentro das Unidades de Terapias } \\
\text { Intensivas, estão relacionados a três categorias: } \\
\text { aos medicamentos; aos registros nos prontuá- } \\
\text { rios e seguimento da SAE. }\end{array}$ \\
\hline $\begin{array}{l}\text { Souza CS, Barlema } \\
\text { JGT, Rocha LP, Bar- } \\
\text { lem ELD, Silva TL, } \\
\text { Neutzlinga BRS./ } \\
2019^{(3)}\end{array}$ & $\begin{array}{c}\text { Cultura de segurança } \\
\text { em unidades de terapia } \\
\text { intensiva: perspectiva dos } \\
\text { profissionais de saúde Rev. } \\
\text { Gaúcha Enferm }\end{array}$ & $\begin{array}{c}\text { Conhecer a cultura de segurança } \\
\text { do paciente em unidades de } \\
\text { terapia intensiva }\end{array}$ & $\begin{array}{c}\text { Emergiram duas categorias: Percepção sobre o } \\
\text { erro e Gestão do erro }\end{array}$ \\
\hline Reis CEP/ 2019(18) & $\begin{array}{c}\text { Protocolo de segurança } \\
\text { do paciente na Unidade } \\
\text { de Terapia Intensiva: a } \\
\text { importância da equipe de } \\
\text { enfermagem Revista Cien- } \\
\text { tífica Multidisciplinar Núcleo } \\
\text { do Conhecimento }\end{array}$ & $\begin{array}{l}\text { Enfatizar a temática da visão do } \\
\text { enfermeiro, sobre os cuidados } \\
\text { e as necessidades do paciente, } \\
\text { principalmente quanto a melho- } \\
\text { ria do processo na qualidade do } \\
\text { cuidado }\end{array}$ & $\begin{array}{c}\text { Foram encontrados } 23 \text { artigos, e foi realizada } \\
\text { leitura exploratória dos mesmos, sendo que } \\
\text { destes } 13 \text { artigos foram excluídos por caracte- } \\
\text { rizarem fuga ao tema }\end{array}$ \\
\hline
\end{tabular}

Foi realizada leitura analítica dos artigos selecionados que possibilitou a organização dos assuntos e a sintetização destas que visou à fixação das ideias essenciais para a solução do problema da pesquisa. Para operacionalizar a pesquisa os achados serão discutidos em categorias.

\section{DISCUSSÃO}

\section{Eventos adversos com maior Taxa de Incidência na UTI}

De acordo com Souza, Alves e Alen$\mathrm{car}^{(11)} \mathrm{em}$ seu estudo constatou-se a ocorrência de 152 eventos adversos nas UTI's. O perfil dos pacientes destacou-se por ser do sexo masculino, adultos jovens, com média de 45 anos. Os principais even- tos adversos identificado foram erros de medicação $(29,6 \%)$, lesão por pressão (21\%), extubação não planejada (17\%), infecções associadas aos cuidados de saúde $(15,13 \%)$, perda de sonda $(9,90 \%)$, entre outros.

Cruz e colaboradores ${ }^{(18)}$ destacam como eventos adversos de maior prevalência na UTI: úlcera por pressão, infecções da corrente sanguínea, flebites, hipotensão arterial, em grande parte de competência da enfermagem. A recomendação principal consiste na comunicação, na capacitação e na conscientização dos profissionais de enfermagem sobre os erros e eventos adversos.

Roque, Tonini e $\mathrm{Melo}^{(2)}$ em sua pesquisa afirmam que os eventos adversos mais prevalentes foram 150 por úlcera por pressão (48,2\%), 17 por dano por manejo de cateteres vasculares (5,3\%), 15 por dano por manejo ventilatório (4,6\%), dois por dano por manejo de cateteres urinários $(0,6 \%)$, um por dano por manejo de cateter gástrico $(0,6 \%)$ e um por hipoglicemia (0,3\%). Dos pacientes, $23,4 \%$ evoluíram para óbito durante o período de internação na UTI, dos 115 pacientes que apresentaram evento adverso, 35,6\% faleceram. Trinta e dois pacientes apresentaram 51 eventos adversos de infecção associada aos cuidados de saúde, correspondendo a uma proporção de $9 \%$. Infecção primária de corrente sanguínea $(6,1 \%)$, pneumonia $(4,7 \%)$ e infecção de acesso vascular central $(3,4 \%)$. 
Fatores relacionados à ocorrência dos Eventos adversos na UTI

Os erros ocorrentes na assistência em saúde são considerados provenientes de falhas individuais e também relacionados ao sistema organizacional da instituição. Visto que podem influenciar direta e indiretamente na qualidade da assistência. Tendo como sugestão de melhoria, cultura não punitiva como forma de discussão dos erros pela gestão de saúde, aderindo à comunicação efetiva, voltada ao aprendizado coletivo, item importante das metas de segurança de saúde a ser utilizado na prática assistencial $^{(3)}$.

Segundo Novaretti et al..$^{(10)}$, quando se trata de unidade de terapia intensiva diversas equipes multiprofissionais estão envolvidas no cuidado prestado ao paciente. $\mathrm{O}$ ambiente hospitalar, em que o paciente é considerado grave, possui características que tornam mais susceptível a erros durante a internação.

De acordo com Minuzzi et al.(13), as falhas e os eventos adversos que acometem o paciente crítico e semicrítico podem ocasionar consequências severas, levando inclusive o paciente a óbito. Pode-se citar todos os processos decorrentes das falhas que, como fatores humanos que está ligado diretamente a segurança do paciente, que o profissional deve estar com sua atenção inteiramente voltada para o paciente.

Minuzzi et al. ${ }^{(13)}$ retrata também que no ambiente hospitalar, podem ocorrer algumas distrações como conversas paralelas e alguns estudos comprovam que o maior índice de eventos adversos está relacionado a administração da medicação incorreta. Está associado também a fatores relacionados ao uso de tecnologia em que a falta de manutenção ocasiona insegurança e ineficiência de todo o processo, e o último fator seria relacionado aos recursos materiais em que costuma ocorrer uma gestão de gastos dos insumos de maneira ineficiente, ocasionando a falta de materiais imprescindíveis para a assistência ao paciente, sendo considerado também um grande fator de risco.

Sabe-se que o número de medicamentos sempre esteve associado à ocorrência
Sabe-se que

o número de

medicamentos

sempre esteve

associado à

ocorrência de

eventos adversos,

principalmente

dentro das UTI,

pois conforme,

para garantir a

sobrevida do

paciente se utiliza

de 5 ou mais

medicamentos, e

consequentemente

aumenta-se o

risco de erros,

representando

98,3\% dos

eventos adversos. de eventos adversos, principalmente dentro das UTI, pois conforme ${ }^{(2)}$, para garantir a sobrevida do paciente se utiliza de 5 ou mais medicamentos, e consequentemente aumenta-se o risco de erros, representando 98,3\% dos eventos adversos.

A sobrecarga de trabalho dos profissionais de saúde está diretamente relacionada ao aumento de eventos adversos em unidades hospitalares, desta forma, os gestores de enfermagem devem estar em contato constante com os gestores de recursos humanos para que possam ser instituídas metas de melhorias para a equipe de saúde. Desenvolvendo estratégias e elaborando práticas seguras que forneçam o suporte necessário para os colaboradores ${ }^{(10)}$. $\mathrm{O}$ objetivo dos gestores, neste âmbito, deve estar voltado a minimizar os impactos relacionados à assistência de enfermagem, reduzindo a sobrecarga de trabalho.

Novaretti e colaboradores ${ }^{(10)}$ acrescenta também que a sobrecarga de trabalho de profissionais de enfermagem deve ser compreendida como uma consequência de diversos fatores, já que o enfermeiro não tem como atividade somente a assistência ao paciente, mas também o treinamento $\mathrm{e}$ capacitação de profissionais de enfermagem, gerenciamento de insumos, articulação com outros profissionais da saúde, promoção da organização, fornecimento de orientações aos pacientes e familiares, promovendo, enfim, a gestão multiprofissional em prol do paciente.

Segundo Grilo et al., ${ }^{(14)}$, os profissionais de enfermagem também podem estar submetidos a altos níveis de estresse, sobrecarga de trabalho, ambiente de trabalho inapropriado e associado a isso a insatisfação e desmotivação profissional. Podendo, desta forma, comprometer a segurança do paciente.

Segundo Silva et al., ${ }^{(15)}$ o transporte intra-hospitalar constitui uma extensão dos cuidados realizados em Unidade de Terapia Intensiva, entretanto os resultados deste estudo evidenciam os riscos aos quais os pacientes estão expostos durante o deslocamento, haja vista que foi verificada a ocorrência de incidentes que tiveram como desfecho um evento adverso. Ressal- 
ta que a maioria dos incidentes encontrados foi relacionado aos equipamentos e os eventos adversos observados foram relativos às alterações fisiológicas.

Para Batista ${ }^{(16)}$ a participação não efetiva de gestores, e demais membros da equipe interdisciplinar são fatores que influenciam na ocorrência de Eventos Adversos, outro fator importante é a carga de trabalho excessiva e o número reduzido da equipe de enfermagem.

Cruz et al ${ }^{(17)}$ destaca que os principais erros e falhas que ocorrem dentro das Unidades de Terapias Intensivas, estão relacionados a três categorias: as medicações, aos registros nos prontuários e ao seguimento da SAE.

Visando à promoção da qualidade da assistência de enfermagem e a segurança do paciente é indispensável estabelecer quais eventos adversos mais recorrentes, o perfil dos pacientes envolvidos e os fatores associados ao dano ${ }^{(2)}$. Para que, através disto, torne-se possível reduzir falhas assistenciais.

\section{Medidas de Prevenção dos Eventos Adversos na UTI}

Visando à prevenção dos eventos adversos é necessário a implementação de medidas institucionais, sendo elas: Capacitação profissional e definição de normas técnicas. Os profissionais de saúde devem, também, notificar os erros para que os indicadores sejam fidedignos, entretanto, alguns profissionais, possuem resistência em aceitar ou reconhecer os próprios erros, gerando índices de subnotificação ${ }^{(11)}$.

É imprescindível o registro completo dos dados referente ao quadro clínico do paciente eletronicamente para promover a continuidade no cuidado, visto que as informações dos pacientes poderão ser visualizadas a qualquer momento pela equipe multiprofissional. Devendo ser realizado registro que facilite entendimento de todos os profissionais, de forma sucinta, objetiva, clara e detalhada, evitando também na ocorrência de eventos adversos. Podendo ser utilizado como estratégia na promoção da qualidade do cuidado, a partir de análise dos indicadores obtidos através dos registros ${ }^{(12)}$.
Vale ressaltar também o protocolo de segurança do

paciente que

deve ser seguido

de acordo com a

portaria no 529 ,

do Ministério da

Saúde (2013),

no ano de 2004,

a Organização

Mundial de Saúde

(OMS) elaborou

com o objetivo

principal de

prevenir danos

aos pacientes.

Estabelecido

assim, as metas

internacionais de

Seguranças do

Paciente ...
Vale ressaltar também o protocolo de segurança do paciente que deve ser seguido de acordo com a portaria $\mathrm{n}^{\circ} 529$, do Ministério da Saúde (2013), no ano de 2004, a Organização Mundial de Saúde (OMS) elaborou com o objetivo principal de prevenir danos aos pacientes. Estabelecido assim, as metas internacionais de Seguranças do Paciente que são:

- Identificar o paciente corretamente;

- Melhorar a eficácia na comunicação;

- Melhorar a segurança para medicamentos de alto risco;

- Eliminar procedimentos errados, no paciente errado;

- Reduzir o risco de infecções hospitalares;

- Reduzir o risco de lesão do paciente resultante de quedas.

De acordo com Reis ${ }^{(18)}$ a forma de comunicação e as abordagens terapêuticas fornecem um suporte indispensável ao cuidado, uma vez que a equipe de enfermagem é a equipe que possui maior convivência com paciente e sua família, ressalta também que a implementação da educação continuada para os profissionais é de grande importância e a qualificação profissional deve ser contínua para que a equipe consiga alcançar seus objetivos e minimizar os impactos direcionados à assistência, fornecendo assistência segura e de qualidade.

\section{CONCLUSÕES}

O presente estudo ressalta a importância dos profissionais de enfermagem na segurança do paciente na Unidade de Terapia Intensiva (UTI), minimizando os eventos adversos e identificando as possíveis causas para que não ocorram. Devem se atentar para que, caso ocorram, sejam notificados e tratados pontualmente. Para prevenção dos eventos adversos, devem ser implementadas, estratégias pelos gestores de enfermagem visando melhoria da assistência, redução de sobrecarga de trabalho dos profissionais, registros completos em prontuários eletrônicos, educação conti- 
nuada, comunicação efetiva entre equipe multiprofissional, estabelecimento de normas institucionais e minimização de falhas assistenciais.

A qualidade da assistência prestada ao paciente engloba também o gerenciamento de riscos institucionais e seus indicadores voltados para princípios e diretrizes com a finalidade de oferecer uma assistência humanizada e segura, ampliando as boas práticas de funcionamento do serviço de saúde e garantindo a qualidade que asseguram o quão os serviços são ofertados com padrões de qualidade.

O presente estudo destaca o sério problema dos eventos adversos na assistência à saúde prestada na terapia intensiva, os fatores de risco associados à incidência de eventos e as medidas de prevenção. Tendo como principal limitação, os registros inadequados dos eventos adversos na UTI que foi identificado através dos dados apresentados nos estudos que embasaram esta pesquisa. -

\section{REFERÊNCIAS}

1. Resolução - RDC N³6, de 25 de julho de 2013. Institui ações para a segurança do paciente em serviços de saúde e dá outras providências. Brasília: Ministério da Saúde, Agência Nacional de Vigilância Sanitária, 2013.

2. Roque KE, Tonini T, Melo ECP. Eventos adversos na unidade de terapia intensiva: impacto na mortalidade e no tempo de internação em um estudo prospectivo. Cad. Saúde Pública, 2016; 32(10)1-15.

3. Souza CS, Barlema JGT, Rocha LP, Barlem ELD, Silva TL, Neutzlinga BRS. Cultura de segurança em unidades de terapia intensiva: perspectiva dos profissionais de saúde. Rev. Gaúcha Enferm., 2019; 40(spe): e20180294,

4. Cavalcante AKCB Rocha RC, Nogueira LT, Avelino FVSD Rocha SS. et al. Cuidado seguro ao paciente: contribuições da enfermagem. Revista Cubana., 2015; 31(4).

5. Duarte SCM, Stipp MAC, Silva MM, Oliveira FT. Eventos adversos e segurança na assistência de enfermagem. Rev. Bras. Enferm. [Internet]. 2015 Feb [cited 2021 Jan 28]; 68(1): 144-154. Available from: http://www.scielo.br/scielo.php?script=sci_arttext\&pid=S0034-71672015000100144\&Ing=en.

6. Mendes KDS, Silveira RC de CP, Galvão CM. Revisão integrativa: método de pesquisa para a incorporação de evidências na saúde e na enfermagem. Texto Context - Enferm. 2008; 17(4):758-64. Doi: 10.1590/S0104-07072008000400018

7. Ogrinc G, Davies L, Goodman D, Batalden P, Davidoff F, Stevens D. Standards for QUality Improvement Reporting Excellence 2.0: revised publication guidelines from a detailed consensus process. J Surg Res. 2016; 200(2):676-82.

8. Brun CN, Zuge SS. Revisão sistemática da literatura: desenvolvimento e contribuição para uma prática baseada em evidências na enfermagem. In: Lacerda MR, Costenaro RGS, organizadoras. Metodologias da pesquisa para a enfermagem e saúde. Porto Alegre: Moriá; 2015. p. 77- 98.

9. Barbosa TP, Oliveira GAA, Lopes MNA, Poletti NAA, Beccaria LM. Práticas assistenciais para segurança do paciente em unidade de terapia intensiva. Acta Paul Enferm. 2014; 27(3):243-8

10. Novaretti MCZ, Santos EV, Quitério LM, Daud-Gallotti RM. Sobrecarga de trabalho da Enfermagem e incidentes e eventos adversos em pacientes internados em UTI. Rev. bras. enferm. [Internet]. 2014 [cited 2021 Jan 28]; 67(5): 692-699.
Available from: http://www.scielo.br/scielo.php?script=sci arttext\&pid=S0034-71672014000500692\&lng=en.

11. Souza RF, Alves AS, Alencar IGM. Eventos adversos na unidade de terapia intensiva. Rev enferm UFPE on line., 12(1):19-27, 2018.

12. Costa TD. Avaliação do cuidado de enfermagem na perspectiva da segurança do paciente em Unidade de Terapia Intensiva: na visão de profissionais, pacientes e familiares, 157f, 2015. Tese doutorado em enfermagem. Universidade Federal do Rio Grande do Norte. Centro de Ciências da Saúde. Programa de Pós-Graduação em Enfermagem. Natal, RN, 2015

13. Minuzzi AP, Salum NC, Locks MOH, Amante LN, Matos E. Contribuições da equipe de saúde visando à promoção da segurança do paciente no cuidado intensivo. Esc. Anna Nery [Internet]. 2016 [cited 2021 Jan 28]; 20(1): 121-129. Available from: http://www.scielo.br/scielo.php?script=sci arttext\&pid=S1414-81452016000100121\&Ing=en. http:// dx.doi.org/10.5935/1414-8145.20160017.

14. Grilo PK, Barbosa RL, Andolhe R, Oliveira EM, Ducci AJ, Bregalda RS et al. Carga de trabalho de enfermagem, estresse/Burnout, satisfação e incidentes em unidade de terapia intensiva de trauma. Texto contexto - enferm. [Internet]. 2017 [cited 2021 Jan 28] ; 26(3): e1720016. Available from: http://www.scielo.br/scielo.php?script=sci_arttext\&pid=S0104-07072017000300322\&Ing=en. Epub Sep 21, 2017. http://dx.doi.org/10.1590/010407072017001720016.

15. Silva R, Amante LN, Salum NC, Girondi JBR, Sebold LF. Incidentes e eventos adversos no transporte intra-hospitalar em terapia intensiva. Revista de Enfermagem do Centro-Oeste Mineiro. 2018; 8: e2805.

16. Batista CF. Fatores que influenciam a ocorrência de eventos adversos Incidentes e eventos adversos no transporte intra hospitalar em terapia intensiva na unidade de terapia intensiva. Rev. Eletr. Evid \& Enferm. 2018; 2(1):15-37.

17. Cruz FF, Gonçalves RP, Raimundo SR, Amaral MS. Segurança do paciente na uti: uma revisão da literatura. Revista Científica FacMais. 2018; 7(1):167-187

18. Reis CEP. Protocolo de segurança do paciente na Unidade de Terapia Intensiva: a importância da equipe de enfermagem. Revista Científica Multidisciplinar Núcleo do Conhecimento. 2019; 4(3):104-113. 